\title{
GLOBAL DYNAMICS OF THE RÖSSLER SYSTEM WITH CONSERVED QUANTITIES
}

\author{
MAURÍCIO FIRMINO SILVA LIMA ${ }^{1}$ AND JAUME LLIBRE ${ }^{2}$
}

\begin{abstract}
The subject of this paper concerns with a class of Rössler systems that admits conserved quantities. For this class of systems a complete description of the global dynamics in the Poincaré sphere is provided.
\end{abstract}

\section{Introduction}

In general it is not easy to detect when a differential system has some kind of integrability. This question can occupy an entire book, see for instance [9]. The integrability of a differential system exhibits some recognizable features such as the existence of conserved quantities, or the presence of algebraic invariants, or the ability to give explicit solutions, see for instance [3].

In this paper we shall work with the notion of integrability associated to conserved quantities, either with the existence of first integrals or conserved quantities independent of the time, or with the existence of invariants or conserved quantities depending on the time. Clearly when a system presents first integrals or invariants, they strongly help to understand the dynamics of the system.

In this paper we study the Rössler system [6]. This system is a three dimensional system given by

$$
\begin{array}{ll}
\dot{x}=-y-z & =P_{1}(x, y, z), \\
\dot{y}=x+a y & =P_{2}(x, y, z), \\
\dot{z}=b-c z+x z & =P_{3}(x, y, z),
\end{array}
$$

where $a, b, c$ are real parameters and the dot denotes the derivative with respect to the independent variable $t$ that we call the time.

The Rössler system is a famous dynamical model studied by many authors. Thus related with this system there are more than 300 papers published (see MathSciNet) in which mainly the dynamical chaos is investigated.

In applications the parameters $a, b$ and $c$ are considered positive real numbers and it is known that this system exhibits, for some values of the parameters, chaotic behavior. On the other hand in [8] (see also [4]) a necessary and sufficient condition for the complete integrability of system (1) is stated. More precisely it is shown that system (1) is completely integrable if and only if $a=b=c=0$ (see Theorem 1 part (iii) of [8]). In this case system (1) becomes

$$
\begin{array}{ll}
\dot{x}=-y-z & =P_{1}(x, y, z), \\
\dot{y}=x & =P_{2}(x, y, z), \\
\dot{z}=x z & =P_{3}(x, y, z) .
\end{array}
$$

2010 Mathematics Subject Classification. 34A36, 34A60, 34C25, 34C37.

Key words and phrases. equilibrium point, first integral, invariant manifolds, periodic orbit, homoclinic orbit, Poincaré compactification, Poincaré sphere, Rössler system. 
This particular Rössler system has a Darboux first integral given by

$$
H_{1}=z e^{-y}
$$

and a polynomial first integral

$$
H_{2}=x^{2}+y^{2}+2 z,
$$

which are functionally independent.

Also in [8] it is shown that if $a=c=0$ and $b \in \mathbb{R}$, then system (1) has

$$
H=x^{2}+y^{2}+2 z-2 b t
$$

as an invariant under the flow (see Theorem 1 part (ii) of [8]).

In what follows we will give a complete description of the dynamics of the integrable Rössler system (2) in the Poincaré sphere using the Poincaré compactification. We also give a description of the phase portrait of system (1) for $a=c=0$ and $b \neq 0$.

First we study the general non integrable system at the infinity that corresponds to analyze the system restricted to the boundary $\mathbb{S}^{2}$ of an open ball diffeomorphic to $\mathbb{R}^{3}$, via the Poincaré compactification.

Second we describe the dynamics of the integrable system in $\mathbb{R}^{3}$ using the expression of the first integrals $H_{1}$ and $H_{2}$. We also describe the asymptotic behavior of the orbits that go to or come from the infinity.

Finally we analyze the behavior of the orbits of system (1) with $a=c=0$ and $b \neq 0$.

The main results of this paper are the following

Theorem 1. On the Poincaré sphere at infinity represented by $\mathbb{S}^{2}$, the differential system (1) has two great circles (the ends of the planes $x=0$ and $z=0$ ) filled of singular points, and these are all the singular points at infinity. Moreover the phase portrait on the infinity sphere $\mathbb{S}^{2}$ is represented in Figure 1.

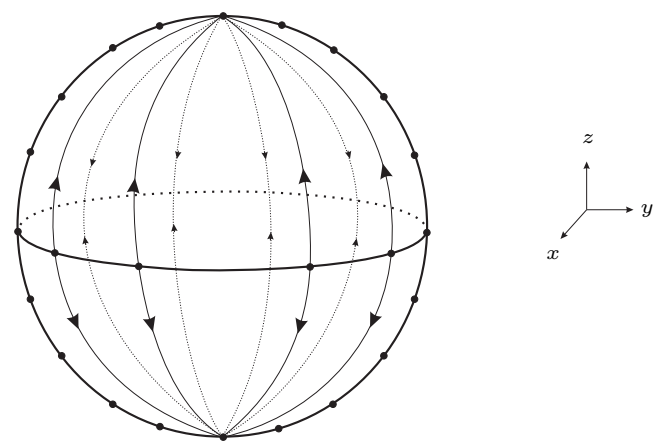

Figure 1. Phase portrait of system (1) on the Poincaré sphere at infinity.

Theorem 1 is proved in section 3 .

Theorem 2. The following statements hold for system (2).

(a) There is a line $(0, y,-y)$ with $y \in \mathbb{R}$ of singular points. Moreover the plane $z=0$ is invariant and on this plane the system has a linear center. 
(b) On the invariant paraboloid $H_{2}=h_{2}$ with $h_{2}>-1$ there is a homoclinic orbit to the equilibrium point $\left(0, y_{1},-y_{1}\right) \in H_{2}^{-1}\left(h_{2}\right)$ with $y_{1}>0$ and a family of periodic orbits connecting this homoclinic orbit with the equilibrium point $\left(0, y_{2},-y_{2}\right) \in H_{2}^{-1}\left(h_{2}\right)$ with $y_{2}<0$, see Figure 2.

(c) The orbits on the invariant paraboloid $H_{2}=h_{2}$ with $h_{2} \leq-1$ are all unbounded and for $h_{2}=-1$ there is an orbit associated to the equilibrium point $(0,1,-1)$ that is of cusp type, see Figure 3 and 4.

(d) Except for two heteroclinic orbits for $h_{2} \geq-1$ connecting the equilibrium point $\left(0, y_{2},-y_{2}\right) \in H_{2}^{-1}\left(h_{2}\right)$ with the equilibrium point $(0,0,-1)$ of the Poincaré ball, all the unbounded orbits of system (2) are homoclinic orbits to the equilibrium point $(0,0,-1)$.

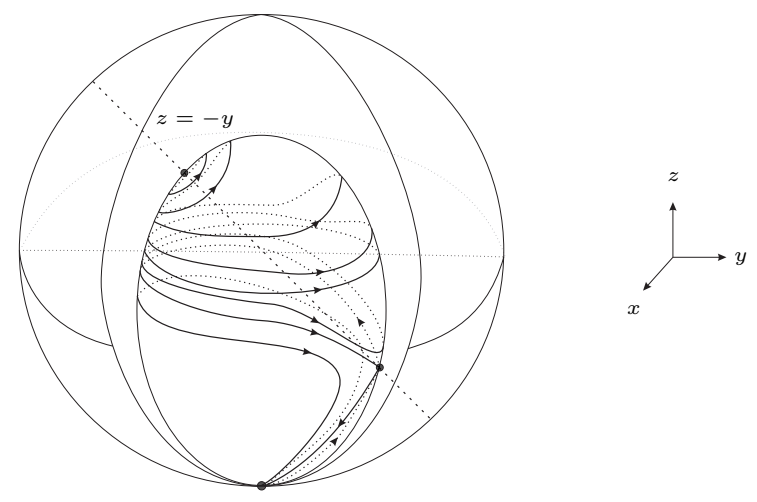

Figure 2. Phase portrait of system (2) on an invariant paraboloid $H_{2}=h_{2}$ with $h_{2}>-1$ inside the Poincaré ball.

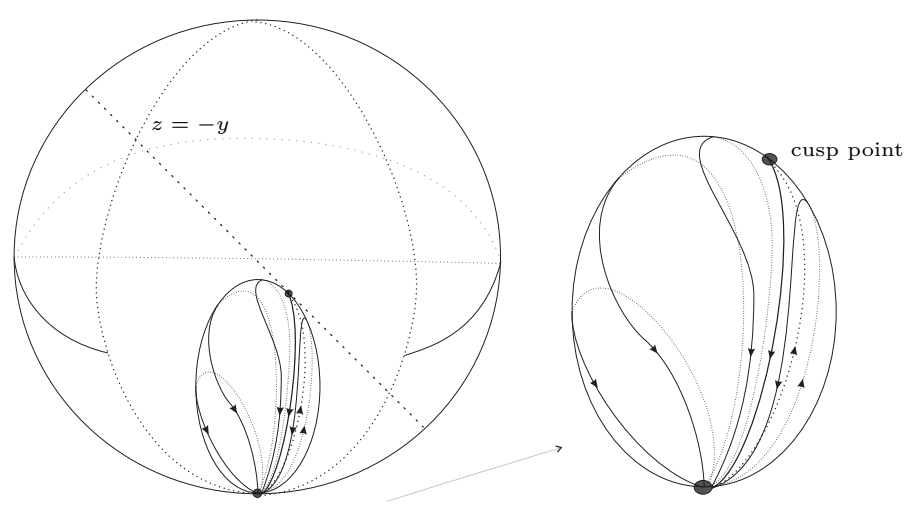

Figure 3. Phase portrait of system (2) on an invariant paraboloid $H_{2}=h_{2}$ with $h_{2}=-1$ inside the Poincaré ball.

Theorem 2 is proved in section 4 


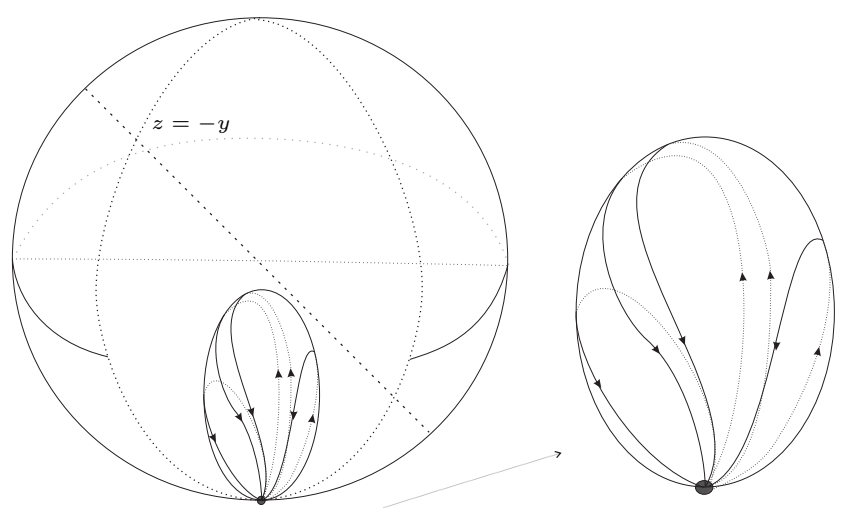

Figure 4. Phase portrait of system (2) on an invariant paraboloid $H_{2}=h_{2}$ with $h_{2}<-1$ inside the Poincaré ball.

Remark 3. Note that in $h_{2}=-1$ qualitatively we see part of the Bogdanov-Takens bifurcation (see [7]) in the neighborhood of the equilibrium point $(0,1,-1)$ varying the parameter $h_{2}$.

Theorem 4. System (1) with $a=c=0$ and $b \neq 0$ has no bounded solution. In fact all the orbits of system (1) go to and come from the infinity in the following way.

(a) If $b>0$ then all the orbits of system (1) go to the Poincaré sphere $\mathbb{S}^{2}$ (i.e. the infinity of $\mathbb{R}^{3}$ ) and come from the point $(0,0,-1) \in \mathbb{S}^{2}$.

(b) If $b<0$ then all the orbits of system (1) go to the point $(0,0,-1) \in \mathbb{S}^{2}$ and come from the Poincaré sphere $\mathbb{S}^{2}$ (i.e. the infinity of $\mathbb{R}^{3}$ ).

In particular, system (1) for $a=c=0$ and $b \neq 0$ has no equilibrium points or periodic orbits.

Theorem 4 is proved in section 5 .

As far as we know all the results of the previous theorems are new, with the exception of statements (a) and (b) of Theorem 2 which were described in $\mathbb{R}^{3}$ without proving them, in [5]. Here they are proved and extended to infinity, i.e., to the Poincaré sphere.

For this purpose we briefly present in section 2 the Poincaré compactification for the particular case considered in this paper, that is the Poincaré compactification in $\mathbb{R}^{3}$.

We must mention that system (2) exhibits a Hamiltonian structure. Moreover, it admits a bi-Hamiltonian structure meaning that there are infinitely different ways of proving this Hamiltonian structure. This result follows from the result of section 5 of [1]. In fact, this result was known before, but in [1] its presentation is very clear. Of course, this Hamiltonian structure cannot be extended to the Poincaré sphere, because there are stable equilibrium points at infinity.

\section{Poincaré COMPACTIFicAtion in $\mathbb{R}^{3}$}

A polynomial vector field $X$ in $\mathbb{R}^{n}$ can be extended to a unique analytic vector field on the sphere $\mathbb{S}^{n}$. The technique for making such an extension is called the Poincaré compactification and allows us to study a polynomial vector field in a 
neighborhood of infinity, which corresponds to the equator $\mathbb{S}^{n-1}$ of the sphere $\mathbb{S}^{n}$. Poincaré introduced this compactification for polynomial vector fields in $\mathbb{R}^{2}$. Its extension to $\mathbb{R}^{n}$ for $n>2$ can be found in [2]. In this section we describe the Poincaré compactification for polynomial vector fields in $\mathbb{R}^{3}$ following closely what is made in [2].

In $\mathbb{R}^{3}$ we consider the polynomial differential system

$$
\dot{x}=P_{1}(x, y, z), \quad \dot{y}=P_{2}(x, y, z), \quad \dot{z}=P_{3}(x, y, z),
$$

or equivalently its associated polynomial vector field $X=\left(P_{1}, P_{2}, P_{3}\right)$. The degree $m$ of $X$ is defined as $m=\max \left(\operatorname{deg}\left(P_{i}\right) ; i=1,2,3\right)$.

Let $\mathbb{S}^{3}=\left\{y=\left(y_{1}, y_{2}, y_{3}, y_{4}\right) \in \mathbb{R}^{4} ;\|y\|=1\right\}$ be the unit sphere in $\mathbb{R}^{4}, \mathbb{S}_{+}=\{y \in$ $\left.\mathbb{S}^{3} ; y_{4}>0\right\}$ and $\mathbb{S}_{-}=\left\{y \in \mathbb{S}^{3} ; y_{4}<0\right\}$ be the northern and southern hemispheres of $\mathbb{S}^{3}$ respectively. The tangent space to $\mathbb{S}^{3}$ at the point $y$ is denoted by $T_{y} \mathbb{S}^{3}$. Then the tangent plane

$$
T_{(0,0,0,1)} \mathbb{S}^{3}=\left\{\left(x_{1}, x_{2}, x_{3}, 1\right) \in \mathbb{R}^{4} ;\left(x_{1}, x_{2}, x_{3}\right) \in \mathbb{R}^{3}\right\}
$$

is identified with $\mathbb{R}^{3}$.

We consider the central projections $f_{+}: T_{(0,0,0,1)} \mathbb{S}^{3} \rightarrow \mathbb{S}_{+}$and $f_{-}: T_{(0,0,0,1)} \mathbb{S}^{3} \rightarrow$ $\mathbb{S}_{-}$defined by $f_{ \pm}(x)= \pm\left(x_{1}, x_{2}, x_{3}, 1\right) / \Delta x$, where $\Delta(x)=\left(1+\sum_{i=1}^{3} x_{i}^{2}\right)^{1 / 2}$. Through these central projection $\mathbb{R}^{3}$ is identified with the northern and southern hemispheres. The equator of $\mathbb{S}^{3}$ is $\mathbb{S}^{2}=\left\{y \in \mathbb{S}^{3} ; y_{4}=0\right\}$. Clearly $\mathbb{S}^{2}$ can be identified with the infinity of $\mathbb{R}^{3}$.

The maps $f_{+}$and $f_{-}$define two copies of $X$ on $\mathbb{S}^{3}$, one $D f_{+} \circ X$ in the northern hemisphere and the other $D f_{-} \circ X$ in the southern one. Denote by $\bar{X}$ the vector field on $\mathbb{S}^{3} \backslash \mathbb{S}^{2}$ which, restricted to $\mathbb{S}_{+}$coincides with $D f_{+} \circ X$ and restricted to $\mathbb{S}_{-}$coincides with $D f_{-} \circ X$.

The expression for $\bar{X}(y)$ on $\mathbb{S}_{+} \cup \mathbb{S}_{-}$is

$$
\bar{X}(y)=y_{4}\left(\begin{array}{ccc}
1-y_{1}^{2} & -y_{2} y_{1} & -y_{3} y_{1} \\
-y_{1} y_{2} & 1-y_{2}^{2} & -y_{3} y_{2} \\
-y_{1} y_{3} & -y_{2} y_{3} & 1-y_{3}^{2} \\
-y_{1} y_{4} & -y_{2} y_{4} & -y_{3} y_{4}
\end{array}\right)\left(\begin{array}{c}
P_{1} \\
P_{2} \\
P_{3}
\end{array}\right) .
$$

where $P_{i}=P_{i}\left(y_{1} /\left|y_{4}\right|, y_{2} /\left|y_{4}\right|, y_{3} /\left|y_{4}\right|\right)$. Written in this way $\bar{X}(y)$ is a vector field in $\mathbb{R}^{4}$ tangent to the sphere $\mathbb{S}^{3}$.

Now we can analytically extend the vector field $\bar{X}(y)$ to the whole sphere $\mathbb{S}^{3}$ by considering $p(X)=y_{4}^{m-1} \bar{X}(y)$, where $m$ is the degree of $X$. This extended vector field $p(X)$ is called the Poincaré compactification of $X$ on $\mathbb{S}^{3}$.

As $\mathbb{S}^{3}$ is a differentiable manifold, in order to compute the expression for $p(X)$ we can consider the eight local charts $\left(\mathcal{U}_{i}, F_{i}\right),\left(\mathcal{V}_{i}, G_{i}\right)$, where $\mathcal{U}_{i}=\left\{y \in \mathbb{S}^{3} ; y_{i}>0\right\}$ and $\mathcal{V}_{i}=\left\{y \in \mathbb{S}^{3} ; y_{i}<0\right\}$ for $i=1,2,3,4$; the diffeomorphisms $F_{i}: \mathcal{U}_{i} \rightarrow \mathbb{R}^{3}$ and $G_{i}: \mathcal{V}_{i} \rightarrow \mathbb{R}^{3}$ for $i=1,2,3,4$ are the inverses of the central projections from the origin to the tangent planes at the points $( \pm 1,0,0,0),(0, \pm 1,0,0),(0,0, \pm 1,0)$ and $(0,0,0, \pm 1)$, respectively. Now we do the computations on $\mathcal{U}_{1}$. Suppose that the origin $(0,0,0,0)$, the point $\left(y_{1}, y_{2}, y_{3}, y_{4}\right) \in \mathbb{S}^{3}$ and the point $\left(1, z_{1}, z_{2}, z_{3}\right)$ in the tangent plane to $\mathbb{S}^{3}$ at $(1,0,0,0)$ are collinear. Then we have $1 / y_{1}=z_{1} / y_{2}=$ $z_{2} / y_{3}=z_{3} / y_{4}$, and consequently $F_{1}(y)=\left(y_{2} / y_{1}, y_{3} / y_{1}, y_{4} / y_{1}\right)=\left(z_{1}, z_{2}, z_{3}\right)$ defines 
the coordinates on $\mathcal{U}_{1}$. As

$$
D F_{1}(y)=\left(\begin{array}{cccc}
-y_{2} / y_{1}^{2} & 1 / y_{1} & 0 & 0 \\
-y_{3} / y_{1}^{2} & 0 & 1 / y_{1} & 0 \\
-y_{4} / y_{1}^{2} & 0 & 0 & 1 / y_{1}
\end{array}\right)
$$

and $y_{4}^{m-1}=\left(z_{3} / \Delta z\right)^{m-1}$, the analytical vector field $p(X)$ becomes

$$
\frac{z_{3}^{m}}{(\Delta z)^{m-1}}\left(-z_{1} P_{1}+P_{2},-z_{2} P_{1}+P_{3},-z_{3} P_{1}\right)
$$

where $P_{i}=P_{i}\left(1 / z_{3}, z_{1} / z_{3}, z_{2} / z_{3}\right)$.

In a similar way we can deduce the expression of $p(X)$ in $\mathcal{U}_{2}$ and $\mathcal{U}_{3}$. These are

$$
\frac{z_{3}^{m}}{(\Delta z)^{m-1}}\left(-z_{1} P_{2}+P_{1},-z_{2} P_{2}+P_{3},-z_{3} P_{2}\right)
$$

where $P_{i}=P_{i}\left(z_{1} / z_{3}, 1 / z_{3}, z_{2} / z_{3}\right)$ in $\mathcal{U}_{2}$, and

$$
\frac{z_{3}^{m}}{(\Delta z)^{m-1}}\left(-z_{1} P_{3}+P_{1},-z_{2} P_{3}+P_{2},-z_{3} P_{3}\right),
$$

where $P_{i}=P_{i}\left(z_{1} / z_{3}, z_{2} / z_{3}, 1 / z_{3}\right)$ in $\mathcal{U}_{3}$.

The expression for $p(X)$ in $\mathcal{U}_{4}$ is $z_{3}^{m+1}\left(P_{1}, P_{2}, P_{3}\right)$ where $P_{i}=P_{i}\left(z_{1}, z_{2}, z_{3}\right)$. The expression for $p(X)$ in the local chart $\mathcal{V}_{i}$ is the same as in $\mathcal{U}_{i}$ multiplied by $(-1)^{m-1}$.

When we work with the expression of the compactified vector field $p(X)$ in the local charts we shall omit the factor $1 /(\Delta z)^{m-1}$. We can do that through a rescaling of the time variable.

We remark that all the points on the sphere at infinity $\mathbb{S}^{2}$ in the coordinates of any local chart have $z_{3}=0$.

In what follows we shall work with the orthogonal projection of $p(X)$ from the closed northern hemisphere to $y_{4}=0$, and we continue denoting this projected vector field by $p(X)$. Note that the projection of the closed northers hemisphere is a closed ball $B$ of radius one, whose interior is diffeomorphic to $\mathbb{R}^{3}$ and whose boundary $\mathbb{S}^{2}$ corresponds to the infinity of $\mathbb{R}^{3}$. Of course $p(X)$ is defined in the whole closed ball $B$ in such a way that the flow on the boundary is invariant. The new vector field on $B$ is called the Poincaré compactification of $X$, and $B$ is called the Poincaré ball, and $\partial B=\mathbb{S}^{2}$ is called the Poincaré sphere at infinity.

\section{Dynamical Behavior of Rössler System at INFinity}

Here we will study the Poincaré compactification of system (1) in the local charts $\mathcal{U}_{i}$ and $\mathcal{V}_{i}$ for $i=1,2,3$ in order to understand the global behavior of the solutions at infinity.

3.1. Local chart $\mathcal{U}_{1}$. Using the results obtained in Section 2 we have that the Poincaré compactification $Z_{1}=p(X)$ of system (1) in the local chart $\mathcal{U}_{1}$ is given by

$$
\begin{aligned}
& \dot{z}_{1}=z_{3}\left(1+z_{1}\left(a-z_{1}+z_{2}\right)\right), \\
& \dot{z}_{2}=z_{2}-\left(c-z_{1}\right) z_{2} z_{3}+z_{2}^{2} z_{3}+b z_{3}^{2}, \\
& \dot{z}_{3}=\left(z_{1}+z_{2}\right) z_{3}^{2} .
\end{aligned}
$$


In the points of the sphere $\mathbb{S}^{2}$ that correspond to the points at infinity we have $z_{3}=0$ and so system (3) becomes

$$
\begin{aligned}
& \dot{z}_{1}=0, \\
& \dot{z}_{2}=z_{2}, \\
& \dot{z}_{3}=0 .
\end{aligned}
$$

The last equation reflects the fact that the infinity $\left(z_{3}=0\right)$ is invariant under the flow. From this system we see that system (1) has at infinity a continuous of singular points given by $\left(z_{1}, 0,0\right)$. Moreover $A_{z_{1}}=D Z_{1}\left(z_{1}, 0,0\right)$ has, for each $z_{1}$, the eigenvalues 1 and 0 with multiplicity 2 . The orbits of the system in the local chart $\mathcal{U}_{1}$ at infinity have the phase portrait given in Figure 5.

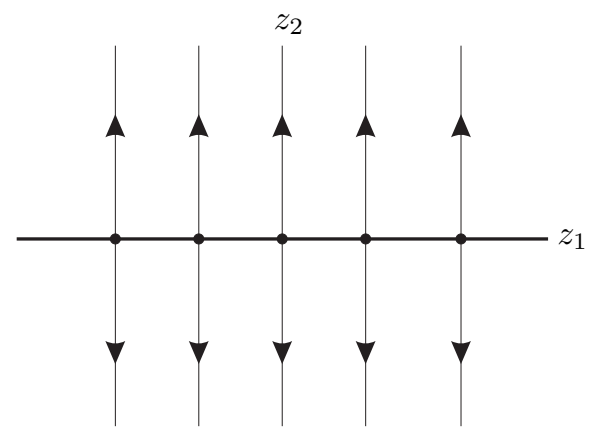

FiguRE 5. The phase portrait in the local chart $\mathcal{U}_{1}$ at infinity.

Note that the equilibria $\left(z_{1}, 0,0\right)$ for $z_{1} \in \mathbb{R}$ represent in the Poincaré sphere $\mathbb{S}^{2}$ part of the equator.

3.2. Local chart $\mathcal{U}_{2}$. In the same way using the results obtained in section 2 the Poincaré compactification $Z_{2}=p(X)$ of system (1) in the local chart $\mathcal{U}_{2}$ is

$$
\begin{aligned}
& \dot{z}_{1}=-z_{1}, \\
& \dot{z}_{2}=z_{1} z_{2}-\left(a+c+z_{1}\right) z_{2} z_{3}+b z_{3}^{2}, \\
& \dot{z}_{3}=-\left(a+z_{1}\right) z_{3}^{2} .
\end{aligned}
$$

In the points of $\mathbb{S}^{2}\left(z_{3}=0\right)$ we have

$$
\begin{aligned}
& \dot{z}_{1}=0, \\
& \dot{z}_{2}=z_{1} z_{2}, \\
& \dot{z}_{3}=0 .
\end{aligned}
$$

Here we have two lines of singular points given by $\left(z_{1}, 0,0\right)$ and $\left(0, z_{2}, 0\right)$ with $z_{1}, z_{2} \in \mathbb{R}$ and the dynamics restricted to $z_{3}=0$ is given in Figure 6 . Moreover $A_{z_{1}}=D Z_{2}\left(z_{1}, 0,0\right)$ has, for each $z_{1}$ the eigenvalues $z_{1}$ and 0 with multiplicity 2 , and $A_{z_{2}}=D Z_{2}\left(0, z_{2}, 0\right)$ has, for each $z_{2}$, the eigenvalue 0 with multiplicity 3 .

As before we observe that the points $\left(z_{1}, 0,0\right)$ with $z_{1} \in \mathbb{R}$ represent half of equator of $\mathbb{S}^{2}$ with endpoints $(1,0,0)$ and $(-1,0,0)$ and the points $\left(0, z_{2}, 0\right)$ with $z_{2} \in \mathbb{R}$ represent half of the great circle connecting the north pole with the south pole. 


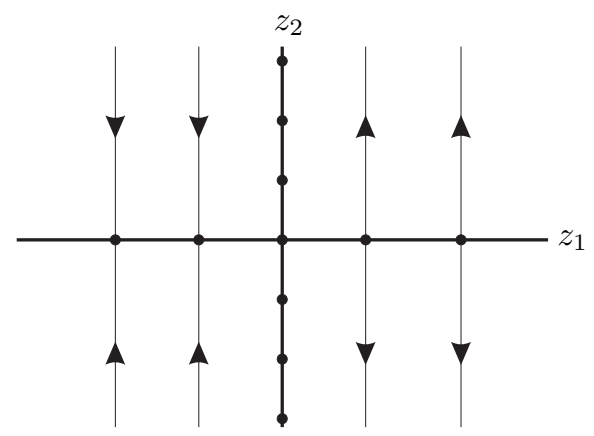

FiguRE 6 . The phase portrait in the local chart $\mathcal{U}_{2}$ at infinity.

3.3. Local chart $\mathcal{U}_{3}$. The Poincaré compactification $Z_{3}=p(X)$ of system (1) in the local chart $\mathcal{U}_{3}$ is

$$
\begin{aligned}
& \dot{z}_{1}=-z_{1}^{2}-z_{3}+c z_{1} z_{3}-z_{2} z_{3}-b z_{1} z_{3}^{2}, \\
& \dot{z}_{2}=z_{1}\left(-z_{2}+z_{3}\right)+z_{2} z_{3}\left(a+c-b z_{3}\right), \\
& \dot{z}_{3}=-z_{3}\left(z_{1}+z_{3}\left(-c+b z_{3}\right)\right) .
\end{aligned}
$$

Restricted to $\mathbb{S}^{2}$ we obtain

$$
\begin{aligned}
& \dot{z}_{1}=-z_{1}^{2}, \\
& \dot{z}_{2}=-z_{1} z_{2}, \\
& \dot{z}_{3}=0,
\end{aligned}
$$

which implies that $Z_{3}$ admits the line $\left(0, z_{2}, 0\right), z_{2} \in \mathbb{R}$ of singular points. These singular points are such that $A_{z_{2}}=D Z_{3}\left(0, z_{2}, 0\right)$ has, for each $z_{2}$ the eigenvalue 0 with multiplicity 3. The phase portrait of $Z_{3}$ restrict to $z_{3}=0$ is given in Figure 7 .

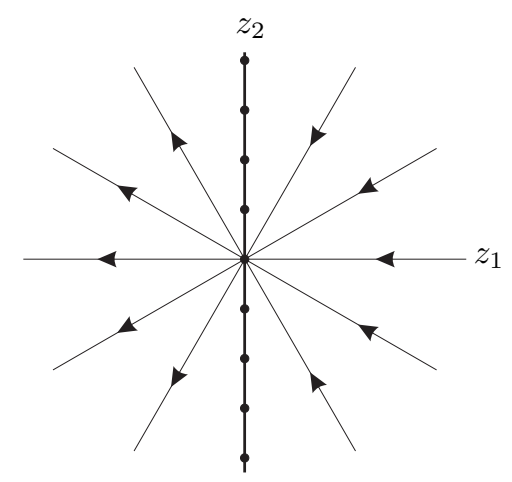

FiguRE 7 . The phase portrait in the local chart $\mathcal{U}_{3}$ at infinity.

Remark 5. We observe that the flows in the $\mathcal{V}_{i}$ charts for $i=1,2,3$ are the same as the ones in the respective $\mathcal{U}_{i}$ charts for $i=1,2,3$ but with the time reversed because the compactified vector field $p(X)$ in $\mathcal{V}_{i}$ coincides with the vector field in $\mathcal{U}_{i}$ multiplied by -1 for each $i=1,2,3$.

From the subsections 3.1, 3.2 and 3.3 we have proved Theorem 1. 
4. Dynamics of Rössler System for the integrable CASE $a=b=c=0$

In this section we describe the dynamics of the Rössler system in $\mathbb{R}^{3}$. We restrict the analysis to the integrable case where the system admits two independent first integrals.

The orbits of the system are given by the intersection of the level surfaces $H_{1}^{-1}\left(h_{1}\right)=\left\{(x, y, z) \in \mathbb{R}^{3} ; H_{1}(x, y, z)=h_{1}\right\}$ and $H_{2}^{-1}\left(h_{2}\right)=\left\{(x, y, z) \in \mathbb{R}^{3} ; H_{2}(x, y, z)=\right.$ $\left.h_{2}\right\}$ where $h_{1}, h_{2} \in \mathbb{R}$.

We observe that $H_{1}=z e^{-y}$ does not depend on $x$. So the graph of the level surface $H_{1}=h_{1}$ is given by the translation of the curve $z=h_{1} e^{y}$ in the $y z$-plane along the $x$-axis. Moreover the level surfaces of $H_{2}=h_{2}$ are paraboloids given by the rotation of the curve $y^{2}+2 z=h_{2}$ in the $y z$-plane around the $z$-axis.

From the above observations to study the intersection between the level surfaces $H_{1}=h_{1}$ and $H_{2}=h_{2}$ it is sufficient to study the intersection between the curves $z e^{-y}=h_{1}$ and $y^{2}+2 z=h_{2}$ in the $y z$-plane.

Proposition 6. Let $C_{h_{1}}^{1}$ and $C_{h_{2}}^{2}$ be the curves $z e^{-y}=h_{1}$ and $y^{2}+2 z=h_{2}$ respectively and suppose that $h_{2} \geq 0$. The relative position between $C_{h_{1}}^{1}$ and $C_{h_{2}}^{2}$ in the $y z$-plane depends on $h_{1}$ and $h_{2}$ in the following way.

(i) If $h_{1}=0$ then there are two points of intersection between $C_{h_{1}}^{1}$ and $C_{h_{2}}^{2}$ if $h_{2}>0$ and one point if $h_{2}=0$ in this case both curves are tangent.

(ii) If $h_{1}^{* *}<h_{1}<0$ then there are three points of intersection between $C_{h_{1}}^{1}$ and $C_{h_{2}}^{2}$.

(iii) If $h_{1}=h_{1}^{* *}$ then there are two points of intersection between $C_{h_{1}}^{1}$ and $C_{h_{2}}^{2}$ and the curves are tangent in one of them.

(iv) If $h_{1}<h_{1}^{* *}$ then there is a unique point of intersection between $C_{h_{1}}^{1}$ and $C_{h_{2}}^{2}$.

(v) If $0<h_{1}<h_{1}^{*}$ then there are two points of intersection between $C_{h_{1}}^{1}$ and $C_{h_{2}}^{2}$.

(vi) If $h_{1}=h_{1}^{*}$ then there is a unique point of intersection between $C_{h_{1}}^{1}$ and $C_{h_{2}}^{2}$. In this case the curves are tangents.

(vii) If $h_{1}>h_{1}^{*}$ then there is no point of intersection between $C_{h_{1}}^{1}$ and $C_{h_{2}}^{2}$.

Here $h_{1}^{*}=\frac{-1+\sqrt{1+h_{2}}}{e^{\left(1-\sqrt{1+h_{2}}\right)}}$ and $h_{1}^{* *}=-\frac{1+\sqrt{1+h_{2}}}{e^{\left(1+\sqrt{1+h_{2}}\right)}}$. The configurations of the possible intersection between the curves is presented in Figures 8 and 9.

Proof. First of all it is easy to see that if $h_{1}=0$ then there are two points of intersection between $C_{h_{1}}^{1}$ and $C_{h_{2}}^{2}$ if $h_{2}>0$ and a unique point if $h_{2}=0$. In this case both curves are tangent at $(0,0)$.

Now suppose that $h_{1}<0$. We study the intersection between $C_{h_{1}}^{1}$ and $C_{h_{2}}^{2}$. So we have to solve the system

$$
z=h_{1} e^{y}, \quad z=\frac{h_{2}-y^{2}}{2} .
$$

Observe that to solve system (4) is equivalent to find the zeroes of the function

$$
f(y)=2 h_{1} e^{y}+y^{2}-h_{2} \quad \text { with } \quad y \in \mathbb{R} .
$$

We have that

(a) $f(0)=2 h_{1}-h_{2}<0$; 
(b) $\lim _{y \rightarrow-\infty} f(y)=\infty$;

(c) $f^{\prime}(y)=2\left(h_{1} e^{y}+y\right)<0$ if $y \leq 0$.

From $(a),(b)$ and $(c)$ we conclude that $f$ has a unique zero in the interval $(-\infty, 0]$. Now for $y \geq 0$ we have

$$
f^{\prime \prime}(y)=2\left(h_{1} e^{y}+1\right)=0 \text { if and only if } y=\log \left(-\frac{1}{h_{1}}\right)=y^{*} .
$$

Moreover

$$
\begin{aligned}
& f^{\prime \prime}(y)>0 \text { if } y \in\left(0, y^{*}\right) \text { and } f^{\prime \prime}(y)<0 \text { if } y \in\left(y^{*}, \infty\right) . \\
& \text { If }-1<h_{1} \leq-\frac{1}{e} \text { we have } y^{*}>0 \text { and } f^{\prime} \text { is } \\
& \text { strictly increasing in the interval }\left(0, y^{*}\right) \text { and } \\
& \text { strictly decreasing in the interval }\left(y^{*}, \infty\right) .
\end{aligned}
$$

Moreover $f^{\prime}(0)=2 h_{1}<0$ and $f^{\prime}\left(y^{*}\right)=2\left(\log \left(-\frac{1}{h_{1}}\right)-1\right) \leq 0$. So $f^{\prime}(y) \leq 0$ for all $y \in(0, \infty)$, and $f$ is decreasing in the interval $(0, \infty)$. As $f(0)<0$ it follows that there is no zero of $f$ in $(0, \infty)$.

If $h_{1} \leq-1$ we have $y^{*} \leq 0$ and $f^{\prime \prime}(y) \leq 0$ for all $y \in(0, \infty)$. A similar argument shows that there is no zero of $f$ in $(0, \infty)$. Consequently there is a unique zero of $f$ in the interval $(-\infty, \infty)$ if $h_{1} \leq-\frac{1}{e}$.

Now suppose $-\frac{1}{e}<h_{1}<0$. In this case $y^{*}>0$. As $f^{\prime}(0)<0$ and $f^{\prime}\left(y^{*}\right)=$ $2\left(\log \left(-\frac{1}{h_{1}}\right)-1\right)>0$. From the fact that $f^{\prime}$ is strictly increasing in $\left(0, y^{*}\right)$, strictly decreasing in $\left(y^{*}, \infty\right)$ and that $\lim _{y \rightarrow \infty} f^{\prime}(y)=\lim _{y \rightarrow \infty} 2\left(h_{1} e^{y}+y\right)=-\infty$, we have that there are $y_{1}, y_{2} \in(0, \infty)$ with $0<y_{1}<y^{*}<y_{2}<\infty$ and $f^{\prime}\left(y_{1}\right)=0=f^{\prime}\left(y_{2}\right)$. Moreover

$$
\begin{aligned}
& f^{\prime}(y)<0 \text { if } y \in\left(0, y_{1}\right) \cup\left(y_{2}, \infty\right) \text { and } \\
& f^{\prime}(y)>0 \text { if } y \in\left(y_{1}, y_{2}\right) .
\end{aligned}
$$

Hence $f$ is strictly decreasing in $\left(0, y_{1}\right) \cup\left(y_{2}, \infty\right)$ and strictly increasing in $\left(y_{1}, y_{2}\right)$.

We also have

$$
f\left(y_{2}\right)=2 h_{1} e^{y_{2}}+y_{2}^{2}-h_{2}=y_{2}^{2}-2 y_{2}-h_{2} .
$$

So if $y_{2}<1+\sqrt{1+h_{2}}$ we have $f\left(y_{2}\right)<0$ and in this case $f(y)<0$ for all $y \in(0, \infty)$. On the other hand if $y_{2}>1+\sqrt{1+h_{2}}$ we have $f\left(y_{2}\right)>0$ and as $f(0)<0$ and $\lim _{y \rightarrow \infty} f(y)=-\infty$, there are two zeroes of $f$ in $(0, \infty)$. The case $y_{2}=1+\sqrt{1+h_{2}}$ implies that $f\left(y_{2}\right)=0$ and that $y_{2}$ is the unique zero of $f$ in $(0, \infty)$. Moreover at this point the curves $C_{h_{1}}^{1}$ and $C_{h_{2}}^{2}$ are tangent.

At the point of tangency we must have

$$
\nabla g_{1}=\lambda \nabla g_{2}
$$

where $g_{1}(y, z)=z-h_{1} e^{y}, g_{2}(y, z)=2 z+y^{2}-h_{2}$ and $\lambda \in \mathbb{R} \backslash\{0\}$.

Solving system (5) we obtain $\lambda=1 / 2, z=-y=-\left(1 \pm \sqrt{1+h_{2}}\right)$. As $y>0$ we take $z=-y=-\left(1+\sqrt{1+h_{2}}\right)$. From equation (5) we obtain

$$
h_{1}=-\frac{1+\sqrt{1+h_{2}}}{e^{1+\sqrt{1+h_{2}}}}=h_{1}^{* *}>-\frac{1}{e} .
$$




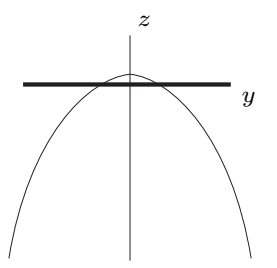

$h_{1}=0$

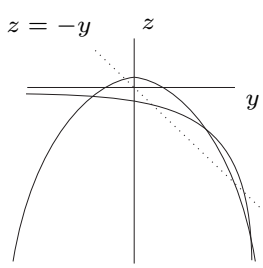

$h_{1}^{* *}<h_{1}<0$

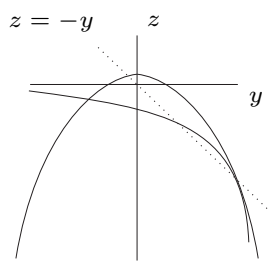

$h_{1}=h_{1}^{* *}$

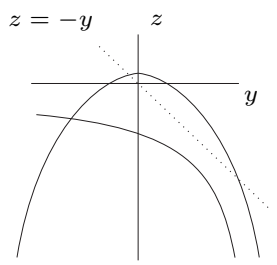

$h_{1}<h_{1}^{* *}$

FiguRE 8 . Relative position between the curves $C_{h_{1}}^{1}$ and $C_{h_{2}}^{2}$ for $h_{2} \geq 0$ and $h_{1} \leq 0$.

Now the case $h_{1}>0$ can be obtained as follows. In the same way that in the previous case if we take the tangency point given by $\left(1-\sqrt{1+h_{2}},-1+\sqrt{1+h_{2}}\right)$ we obtain $h_{1}=h_{1}^{*}=\frac{-1+\sqrt{1+h_{2}}}{e^{1-\sqrt{1+h_{2}}}}$, and in this case there is a unique zero of the function $f$. Also if $0<h_{1}<h_{1}^{*}$ there are two zeroes of the function $f$, and if $h_{1}>h_{1}^{*}$ there is no zero of $f$ (see Figure 9). This concludes the proof.

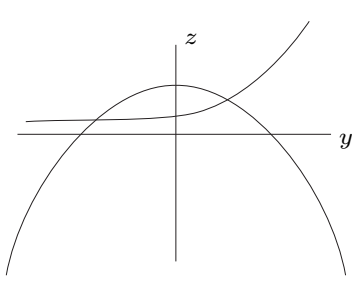

$0<h_{1}<h_{1}^{*}$

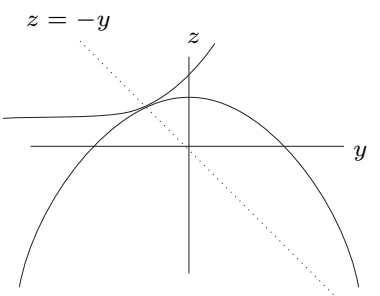

$h_{1}=h_{1}^{*}$

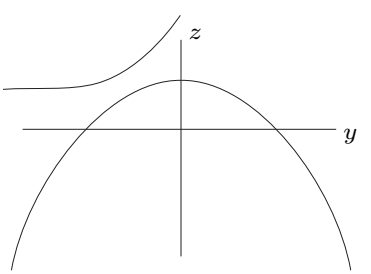

$h_{1}>h_{1}^{*}$

FIGURE 9. Relative position between the curves $C_{h_{1}}^{1}$ and $C_{h_{2}}^{2}$ for $h_{2} \geq 0$ and $h_{1}>0$.

Proposition 7. Suppose that $h_{2}<0$. The relative position between $C_{h_{1}}^{1}$ and $C_{h_{2}}^{2}$ in the yz-plane depends on $h_{1}$ and $h_{2}$ in the following way.

(i) If $h_{1} \geq 0$ then $C_{h_{1}}^{1} \cap C_{h_{2}}^{2}=\emptyset$.

(ii) If $h_{1}<0$ and $2 h_{1}<h_{2}$ then

(ii.a) there are three points of intersection between $C_{h_{1}}^{1}$ and $C_{h_{2}}^{2}$ if $h_{1}>h_{1}^{* *}$;

(ii.b) there are two points of intersection between $C_{h_{1}}^{1}$ and $C_{h_{2}}^{2}$ if $h_{1}=h_{1}^{* *}$ and they are tangent in one of them;

(ii.c) there is a unique point of intersection between $C_{h_{1}}^{1}$ and $C_{h_{2}}^{2}$ if $h_{1}<h_{1}^{* *}$.

(iii) If $h_{1}<0$ and $2 h_{1} \geq h_{2}$ then

(iii.a) there are three points of intersection between $C_{h_{1}}^{1}$ and $C_{h_{2}}^{2}$ if $h_{1}>h_{1}^{*}$;

(iii.b) there are two points of intersection between $C_{h_{1}}^{1}$ and $C_{h_{2}}^{2}$ if $h_{1}=h_{1}^{*}$ and they are tangent in one of them; 
(iii.c) there is a unique point of intersection between $C_{h_{1}}^{1}$ and $C_{h_{2}}^{2}$ if $h_{1}<h_{1}^{*}$. The configurations of the possible intersections between the curves is presented in Figures 10 and 11.

Proof. As in the case $h_{2} \geq 0$ we will study the zeroes of the function $f(y)=$ $2 h_{1} e^{y}+y^{2}-h_{2}$. However first we consider a trivial case when $h_{1} \geq 0$. In this case it is easy to see that $\left\{H_{1}=h_{1}\right\} \cap\left\{H_{2}=h_{2}\right\}=\emptyset$. This means that $C_{h_{1}}^{1} \cap C_{h_{2}}^{2}=\emptyset$. So from now on we will restrict the analysis to the case $h_{1}<0$.

Case 1: Suppose that $h_{2}>-1$. Here we will divide the study into two subcases.

Subcase 1.1: $2 h_{1}<h_{2}$. In this case we have

(i) $f(0)=2 h_{1}-h_{2}<0$;

(ii) $\lim _{y \rightarrow-\infty} f(y)=\infty$;

(iii) $f^{\prime}(y)=2\left(h_{1} e^{y}+y\right)<0$ if $y \leq 0$.

So there is a unique zero of $f$ for $y \leq 0$. A similar analysis as the one done in the previous proposition shows that the possible configuration of the curves $C_{h_{1}}^{1}$ and $C_{h_{2}}^{2}$ are given in Figure 10, where the tangency point occurs when $h_{1}=-\frac{1+\sqrt{1+h_{2}}}{e^{1+\sqrt{1+h_{2}}}}=$ $h_{1}^{* *}$. Hence

if $h_{1}<h_{1}^{* *}$ there is no zero of $f$ for $y>0$;

if $h_{1}=h_{1}^{* *}$ there is a unique zero of $f$ for $y>0$;

if $h_{1}^{* *}<h_{1}<h_{2} / 2$ there are two zeroes of $f$ for $y>0$.

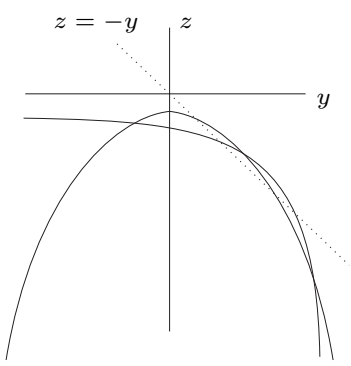

$h_{1}^{* *}<h_{1}$

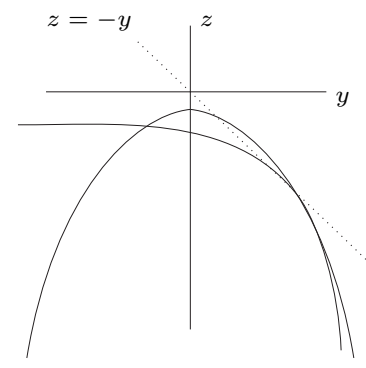

$h_{1}=h_{1}^{* *}$

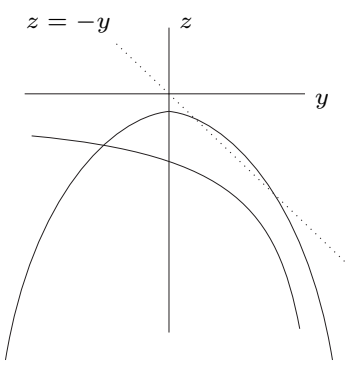

$h_{1}<h_{1}^{* *}$

FIgURE 10. Relative position between the curves $C_{h_{1}}^{1}$ and $C_{h_{2}}^{2}$ for $h_{2}<0$ and $h_{1}<0$. In this three pictures $h_{1}<h_{2} / 2$

Subcase 1.2: $2 h_{1} \geq h_{2}$. In this case

$$
\begin{aligned}
& f(0) \geq 0 ; \\
& \lim _{y \rightarrow-\infty} f(y)=\infty ; \\
& f^{\prime}(y)<0 \text { if } y \leq 0 .
\end{aligned}
$$

So there is no zero of $f$ in $(-\infty, 0)$.

Now for $y \geq 0$ we have $f(0) \geq 0$ and $\lim _{y \rightarrow \infty} f(y)=-\infty$. This implies that there is at least one zero of $f$ in $(0, \infty)$. Moreover

$$
f^{\prime}(0)=2 h_{1}<0
$$


$f^{\prime \prime}(y)=2\left(h_{1} e^{y}+1\right)=0$ if and only if $y=\log \left(-\frac{1}{h_{1}}\right)=y^{*}$ with $f^{\prime \prime}(0)=$ $2\left(h_{1}+1\right)>0$.

As $h_{1} \geq-1 / 2$ we have $y^{*}>0$ and as before $f^{\prime}$ is strictly increasing in $\left(0, y^{*}\right)$ and strictly decreasing in $\left(y^{*}, \infty\right)$. A similar analysis to the one done in the proof of Proposition 6 shows that if $-1 / 2 \leq h_{1} \leq-1 / e$ there is a unique zero of $f$ in $(0, \infty)$ and if $-1 / e<h_{1}<0$ we have $y_{1}, y_{2}>0$ such that $f^{\prime}\left(y_{1}\right)=0=f^{\prime}\left(y_{2}\right)$ and

there is a unique zero of $f$ in $(0, \infty)$ if $f\left(y_{1}\right)>0$;

there are two zeroes of $f$ in $(0, \infty)$ if $f\left(y_{1}\right)=0$ and the curves are tangent in one of them;

there are three zeroes of $f$ in $(0, \infty)$ if $f\left(y_{1}\right)<0$ and $f\left(y_{2}\right)>0$.

In short we have the configuration presented in Figure 11.

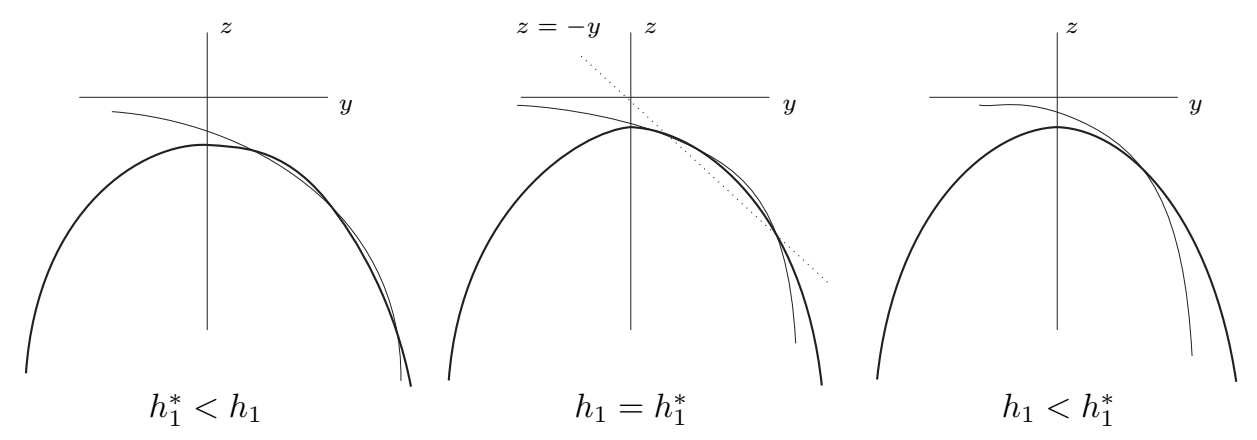

FiguRE 11. Relative position between the curves $C_{h_{1}}^{1}$ and $C_{h_{2}}^{2}$ for $h_{2}<0$ and $h_{1}<0$. In this three pictures $h_{1} \geq h_{2} / 2$

Case 2: Suppose that $h_{2} \leq-1$. In this case $f^{\prime \prime}(0) \leq 0$ and $y^{*} \leq 0$. Consequently $f^{\prime \prime}(y) \leq 0$ for all $y>0$, and $f^{\prime}$ is decreasing in $(0, \infty)$ and as $f^{\prime}(0)<0$ we obtain $f$ decreasing in $(0, \infty)$. This implies from the fact that $f(0) \geq 0$ and $\lim _{y \rightarrow \infty}=-\infty$ that there exists a unique zero of $f$ in $(0, \infty)$. Moreover there is no zero of $f$ in $(-\infty, 0)$. So there exists a unique zero of $f$ in $(-\infty, \infty)$. This configuration coincides with the one in the last picture in Figure 11. We also observe that if $h_{2}=-1$ then the graph of $z=\frac{h_{2}-y^{2}}{2}$ is tangent to the line $z=-y$ and if $h_{2}<-1$ it does not intersect $z=-y$. This concludes the proof.

Now we are in condition to prove Theorem 2.

Proof of Theorem 2. The proof of statement (a) is immediate. The statements (b) and (c) are consequence of Propositions 6 and 7.

First of all observe that the curve $C_{h_{2}}^{2}$ has

(i) two points of intersection with the line $z=-y$ if $h_{2}>-1$;

(ii) one point of intersection with the line $z=-y$ if $h_{2}=-1$. In this case both curves are tangent at the point $(1,-1)$;

(iii) none point of intersection with the line $z=-y$ if $h_{2}<-1$. 
Now note that the points of tangency between $C_{h_{1}}^{1}$ and $C_{h_{2}}^{2}$ (when they exist) occur at $(y,-y)$ with $y \in \mathbb{R}$. They are associated with the equilibria $(0, y,-y), y \in$ $\mathbb{R}$ of system (2). The intersection $\left\{H_{1}=h_{1}\right\} \cap\left\{H_{2}=h_{2}\right\}$ containing the point $(0, y,-y)$ with $y>1$ is formed by a closed curve and two others no limited curves. For each $y>1$ the closed curve corresponds to a homoclinic orbit and the no limited ones to separatrices of the equilibrium.

On the other hand when the intersection of $C_{h_{1}}^{1}$ and $C_{h_{2}}^{2}$ is not tangent we have two possibilities: either the curves are secant in a unique point and the corresponding intersection between the level curves represent a no limited solution of system (2), or the curves intersect into two points that corresponds to a periodic solution. Note that in this case we have a family of periodic solutions connecting the singular point $(0, y,-y)$ with $y<1$ with a homoclinic solution to the equilibrium point $(0, y,-y)$ with $y>1$.

We also observe that there are two curves (for $h_{1}=-1 / e$ and $h_{2}=-1$ ) intersecting just at the point $(1,-1)$. This intersection point is associated with a no limited solution of system (2) of cusp type.

The rest of the proof of the statements (b) and (c) is obtained from the results stated in Propositions 6 and 7. See Figures 8-11.

Finally we prove statement (d) that describes the behavior of the unbounded orbits at infinity. To prove this it is sufficient to observe that the invariant paraboloids $H_{2}=h_{2}$ reach the infinify at the point $(0,0,-1)$ of the boundary of the Poincaré sphere $\mathbb{S}^{2}$.

Observe that in the local chart $\mathcal{V}_{3}$ the paraboloid $x^{2}+y^{2}+2 z=h_{2}$ takes the form

$$
\frac{z_{1}^{2}}{z_{3}^{2}}+\frac{z_{2}^{2}}{z_{3}^{2}}+2 \frac{1}{z_{3}}=h_{2}, \quad \text { or } \quad z_{1}^{2}+z_{2}^{2}+2 z_{3}=h_{2} z_{3}^{2}
$$

At infinity (that corresponds to $z_{3}=0$ ) we have $z_{1}^{2}+z_{2}^{2}=0$, that implies that $z_{1}=z_{2}=0$. So the surface $H_{2}=h_{2}$ for each $h_{2} \in \mathbb{R}$ reaches the infinity at the origin of the local chart $\mathcal{V}_{3}$, that corresponds to the point $(0,0,-1)$ of the Poincaré sphere $\mathbb{S}^{2}$. As all solutions are contained in $H_{2}=h_{2}$ for some $h_{2} \in \mathbb{R}$ we obtain the result.

\section{Dynamical Behavior of Rössler system for $a=c=0$ AND $b \neq 0$}

In this section we study the dynamical behavior of the orbits of Rössler system for the case where the system admits a time dependent function that is invariant under the flow. More precisely we give the proof of Theorem 4.

Proof of Theorem 4. We know that $H$ is an invariant for the system. So given an orbit $\varphi(t)=(x(t), y(t), z(t))$ we have $H(\varphi(t))=\alpha$ for all $t \in I_{\varphi}$, where $I_{\varphi}$ is the maximal interval of definition of $\varphi$ and $\alpha$ is a constant real number.

Consider $p(X)$ the Poincaré compactification of system (1) with $a=c=0$ and $b \neq 0$, and $Z_{3}$ its representation in the local chart $\mathcal{U}_{3}$. So in this chart we have $\varphi(t)=\left(z_{1}(t), z_{2}(t), z_{3}(t)\right)$ and

$$
H\left(z_{1}, z_{2}, z_{3}, t\right)=\frac{z_{1}^{2}}{z_{3}^{2}}+\frac{z_{2}^{2}}{z_{3}^{2}}+\frac{2}{z_{3}}-2 b t
$$


Suppose $b>0$. As $p(X)$ is a vector field defined in a compact manifold it follows that the $I_{\varphi}=(-\infty, \infty)$. Moreover we have

$$
\frac{z_{1}(t)^{2}+z_{2}(t)^{2}+2 z_{3}(t)}{z_{3}(t)^{2}}=\alpha+2 b t \rightarrow \infty \text { when } \quad t \rightarrow \infty .
$$

Since the family of paraboloids

$$
\frac{z_{1}^{2}+z_{2}^{2}+2 z_{3}}{z_{3}^{2}}=k
$$

tends to $\mathbb{S}^{2}$ (i.e. to infinity) when $k \rightarrow \infty$, from (6) it follows that the orbit $\varphi(t)$ crosses this family of paraboloids tending to infinity when $t \rightarrow \infty$.

Since the family of paraboloids $(7)$ tends to the point $(0,0,-1)$ of $\mathbb{S}^{2}$ when $k \rightarrow-\infty$, from $(6)$ we get that the orbit $\varphi(t)$ tends to the point $(0,0,-1)$ when $t \rightarrow-\infty$.

Assume now $b<0$. Then, similar arguments to the case $b>0$ show that the orbit $\varphi(t)$ tends to the point $(0,0,-1)$ of $\mathbb{S}^{2}$ when $t \rightarrow \infty$ and tends to $\mathbb{S}^{2}$ (i.e. to infinity) when $t \rightarrow-\infty$.

\section{ACKNOWLEDGMENTS}

We thank to the referees their comments which allow us to improve the presentation of this paper.

The first author is partially supported by CNPq grant number 200293/2010-9. The second author is partially supported by MCYT/FEDER grant MTM200803437, MICIIN grant number 2009SGR-410 and by AGAUR Academia. Both authors are also supported by the joint project CAPES-MECD grant PHB-2009-0025PC.

\section{REFERENCES}

[1] G.B. Byrnes, F.A. Haggar And G.R.W. Quispel, Sufficient conditions for dynamical systems to have pre-symplectic or pre-implectic structure. Physica A 272, (1999), 99-129.

[2] A. Cima and J. Llibre, Bounded polynomial vector fields. Trans. Amer. Math. Soc. 318, (1990), 557-579.

[3] A. GorIELY, Integrability and Nonintegrability of Dynamical Systems, Advanced Series in Nonlinear Dynamics 19, World Scientific, Singarupe, 2001.

[4] J. Llibre And C. VAlls, Formal and analytic integrability of the Rössler system. Int. J. Bif. and Chaos 17, $n^{\circ} 9$ (2007), 3289-3293.

[5] J. Llibre And X. ZhAng, Darboux integrability for the Rössler system. Int. J. Bif. and Chaos 12, $n^{\circ} 2$ (2002), 421-428.

[6] E. O. Rössler, An equation for continuous chaos. Phys. Lett. A 57, (1976), 397-398.

[7] S. Wiggins, Introduction to Applied Nonlinear Dynamical Systems and Chaos. Springer Texts in Applied Mathematics 2, Second ed., Springer-Verlag, New York, 2003.

[8] X. Zhang, Exponential factors and Darboux integrability for the Rössler system. Int. J. Bif. and Chaos. 14, $n^{\circ} 12$, (2004), 4275-4283.

[9] V. E. Zakharov, What is Integrability? Springer Series in Nonlinear Dynamics, SpringerVerlag, Berlin, 1976.

1 Centro de Matemática ComputaÇão e CogniÇão. Universidade Federal do abC, 09210-170. SAnto AndRE. S.P. BrazIL

E-mail address: mauricio.lima@ufabc.edu.br

2 Departament de Matemàtiques. Universitat Autònoma de Barcelona, 08193 Bellaterra. Barcelona, Catalonia, Spain

E-mail address: jllibre@mat.uab.cat 